\title{
DA OCORRÊNCIA EM VIDA À MORTE: PADRÕES ESPACIAIS DA VIOLÊNCIA CONTRA A MULHER EM PERNAMBUCO
}

\section{FROM OCCURRENCE IN LIFE TO DEATH: SPATIAL PATTERNS OF VIOLENCE AGAINST WOMEN IN PERNAMBUCO}

\author{
Emylle Thais Melo dos Santos \\ ORCID: 0000-0002-4167-965X \\ Programa de Pós-graduação em Saúde Coletiva. \\ Universidade Federal de Pernambuco, Recife, Pernambuco, Brasil. \\ emylle.saantos@hotmail.com \\ Conceição Maria de Oliveira \\ ORCID: 0000-0002-2220-5782 \\ Secretaria de Saúde, Recife, Pernambuco, Brasil \\ coliveira@recife.pe.gov.br
}

Dayane da Rocha Pimentel ORCID: 0000-0002-9863-134X Pós-graduação em Saúde Pública. Instituto Aggeu Magalhães. Fundação Oswaldo Cruz. Recife-Pernambuco, Brasil day pimentel@hotmail.com

Sheyla Carvalho de Barros ORCID: 0000-0001-5399-0296 Secretaria Estadual de Saúde, Pernambuco, Brasil sheyla.barross@gmail.com

Amanda Priscila de Santana Cabral Silva ORCID: 0000-0003-2337-9925 Centro Acadêmico de Vitória. Universidade Federal de Pernambuco, Vitória de Santo Antão, Pernambuco, Brasil. Departamento de Saúde Coletiva. Instituto Aggeu Magalhães. Fiocruz Pernambuco, Recife, Pernambuco, Brasil. amandapscabral@gmail.com

\author{
Cristine Vieira do Bonfim \\ ORCID: 0000-0002-4495-9673 \\ Fundação Joaquim Nabuco, Recife, Pernambuco, Brasil. \\ Programa de Pós-graduação em Saúde Coletiva. \\ Universidade Federal de Pernambuco, Recife, Pernambuco, Brasil. \\ cristine.bonfim@uol.com.br
}

\begin{abstract}
RESUMO
Objetivo: Analisar os padrões espaço-temporais da violência não letal e letal contra a mulher no estado de Pernambuco, no período de 2016 a 2019. Métodos: Estudo ecológico misto com dados de notificação de violência contra mulher registrado no Sistema de Informação de Agravos de Notificação, de homicídios do Sistema de Informações sobre Mortalidade e de feminicídios do Sistema de Informações Policiais. As taxas de violência contra mulher, de homicídios e feminicídios e por ano e município foram normalizadas pela população feminina a cada ano e multiplicado por 100 mil. Foram avaliadas as tendências temporais de cada indicador utilizando o método dos mínimos quadrados ordinários (regressão linear simples) para estimar retas de tendência. Resultados: $\mathrm{Na}$ análise da taxa de violência contra mulher, observou-se que 142 (76,8\%) dos municípios apresentaram tendência crescente, com predomínio na mesorregião do Agreste. Observou-se que 83 $(44,9 \%)$ e $113(61,1 \%)$ dos municípios tiveram tendência estacionária para os homicídios femininos e feminicídios, respectivamente. Conclusão: evidencia-se a necessidade de melhorias nas políticas públicas do estado de Pernambuco que visam à proteção das mulheres em situação de violência, bem como a ampliação dos serviços especializados para as áreas prioritárias.
\end{abstract}

Palavras-chave: Violência contra mulher. Homicídio. Análise espacial. Estatísticas Vitais. Mortalidade.

Recebido em: 25/09/2020

Aceito para publicação em: 13/06/2021. 
Da ocorrência em vida à morte: padrões espaciais da violência contra a mulher em Pernambuco
Emylle Thais Melo dos Santos

Conceição Maria de Oliveira

Dayane da Rocha Pimentel

Sheyla Carvalho de Barros

Amanda Priscila de Santana Cabral Silva

Cristine Vieira do Bonfim

\begin{abstract}
Objective: To analyze the spatiotemporal patterns of non-lethal and lethal violence against women in the state of Pernambuco, from 2016 to 2019. Methods: Mixed ecological study with data on notification of violence against women registered in the Diseases Information System of Notification of homicides from the Mortality Information System and of femicide from the Police Information System. The rates of violence against women, homicides and feminicides and per year and municipality were normalized by the female population each year and multiplied by 100,000 . The temporal trends of each indicator were evaluated using the method of ordinary least squares (simple linear regression) to estimate trend lines. Results: In the analysis of the rate of violence against women, it was observed that 142 $(76.8 \%)$ of the municipalities showed an increasing trend, with a predominance in the mesoregion of Agreste. It was observed that $83(44.9 \%)$ and $113(61.1 \%)$ of the municipalities had a stationary trend towards female and female homicides, respectively. Conclusion: there is an evident need for improvements in public policies in the state of Pernambuco aimed at protecting women in situations of violence, as well as the expansion of specialized services for priority areas.
\end{abstract}

Keywords: Violence Against Women. Homicide. Spatial Analysis. Vital Statistics. Mortality.

\title{
INTRODUÇÃO
}

A ocorrência da violência contra a mulher é histórica e universal, presente em todos os países e grupos sociais, independente das condições socioeconômicas e culturais (WHO, 2021). Trata-se de um dos problemas sociais e de saúde pública mais abrangentes e subestimados do mundo (WHO, 2021). As violações são definidas como qualquer ato ou comportamento cometido por parceiro, família ou comunidade que cause danos físicos, psicológicos ou sexuais a mulher (MENEGHEL; PORTELLA, 2017). As agressões podem ocorrer de diversas formas, com variados graus de severidade, e não acontece isoladamente, faz parte de uma sequência de episódios crescentes, que pode ter como desfecho o feminicídio (LEITE et al., 2019, PENGPID et al., 2018). No mundo, estimase que $30 \%$ de todas as mulheres já tenham sofrido violência física ou sexual (WHO, 2021).

Globalmente são estimados 66.000 homicídios intencionais de mulheres ao ano, equivalente a $17 \%$ do total de mortes por agressão, com concentração nas regiões do Caribe, América Central e na América do Sul (MARGARITES; MENEGHEL; CECCON, 2017). Em 2015, o Brasil ocupou o $5^{\circ}$ lugar no ranking de 83 países nos homicídios de mulheres (WAISELFISZ, 2015). Quando comparada a outros países, a taxa de homicídios de mulheres brasileiras é 48 vezes maior que a do Reino unido, 24 vezes mais que Irlanda e 16 vezes mais que o Japão (WAISELFISZ, 2015).

O feminicídio é conceituado como a morte intencional de uma mulher em decorrência do seu sexo, ou seja, pelo fato de ser mulher (GOMES, 2018; SANZ-BARBERO; BARÓN; VIVES-CASES, 2019). Esse fenômeno é sensível a estruturas patriarcais machistas permeadas por relações desiguais de gênero e marginaliza o exercício de direitos humanos fundamentais (FERNÁNDEZ-GONZÁLEZ; ORUE, 2017, ILANZO et al. 2018). No ano de 2015 foi instituída a Lei 13.104/2015, considerando o feminicídio como circunstância qualificadora quando o homicídio envolver a questão de gênero, confrontando o assassinato de mulheres, com fixação de penas mais elevadas a tais eventos (12 a 30 anos de reclusão) (BRASIL, 2015).

No Brasil, grande parte das notificações de violência contra a mulher registradas nos serviços de saúde é perpetrada por parceiro íntimo, com destaque para a violência física, psicológica e sexual (MASCARENHAS et al., 2020). Outra pesquisa conduzida no país, no período de 2011 a 2016, com 800.000 notificações de violência contra mulheres e 16.500 mortes associadas, identificou um risco de mortalidade oito vezes maior para as mulheres expostas à violência, quando comparado com a população feminina em geral (SANDOVAL et al., 2020). A mesma pesquisa estimou que 100 mulheres morrem semanalmente como consequência direta ou indireta da exposição à violência no país (SANDOVAL et al., 2020). Entre 2012 e 2017, a taxa de homicídio por 100 mil mulheres passou de 4,6 para 4,7 com predomínio nas regiões Norte e Nordeste. No ano de 2017, o estado de Pernambuco apresentou uma taxa de 6,3 homicídios por 100 mil mulheres, registro superior a 15 unidades federativas (IPEA, 2019). 
O emprego de técnicas espaciais possibilita reconhecer áreas que apresentam maior risco de ocorrência de feminicídios (SILVA et al. 2019). Recentemente, em estudos que discutem questões relativas à violência contra a mulher vem sendo adotado como unidade de análise o território (INGRAM e COSTA, 2016; OLIVEIRA et al., 2019; SAN SEBASTIÁN et al., 2019). Diversas abordagens geoespaciais identificaram áreas com maior vulnerabilidade à violência doméstica contra mulher nos estados da Paraíba e Rondônia (LUCENA et al., 2012; RIBEIRO et al., 2015). No Paraná aplicou-se a análise espacial para verificar as disparidades regionais na violência sexual cometida pelo parceiro íntimo contra as mulheres, com a localização de áreas e clusters de maior risco (FONTES et al., 2018).

Assim, além de viabilizar a compreensão dos determinantes em saúde, o uso dessa metodologia possibilita priorizar zonas vulneráveis e subsidiar as tomadas de decisão em serviços intersetoriais responsáveis pela prevenção social da violência (BARBOSA et al., 2019). Nessa perspectiva, o estudo teve como objetivo analisar os padrões espaço-temporais da violência não letal e letal contra a mulher no estado de Pernambuco.

\section{MÉTODOS}

Trata-se de um estudo ecológico misto realizado no estado de Pernambuco, no período de 2016 a 2019. Este recorte temporal justifica-se pelo fato da qualificadora feminicídio ter entrado em vigor em março de 2015 (Lei no 13.104/2015) e, com isso, os dados da Secretaria de Defesa Social, somente estavam disponíveis a partir de 2016. O ano de 2019 é o mais recente com dados disponíveis nas três bases analisadas.

O estado possui uma extensão territorial de $98.312 \mathrm{~km}^{2}$, é composto por 184 municípios e o distrito estadual de Fernando de Noronha, distribuídos por cinco mesorregiões (Sertão, São Francisco, Agreste, Mata e Metropolitana do Recife) e 12 Regiões de Saúde (Figura 1).

Figura 1 - Desenho da regionalização do estado de Pernambuco por mesorregiões (2011).

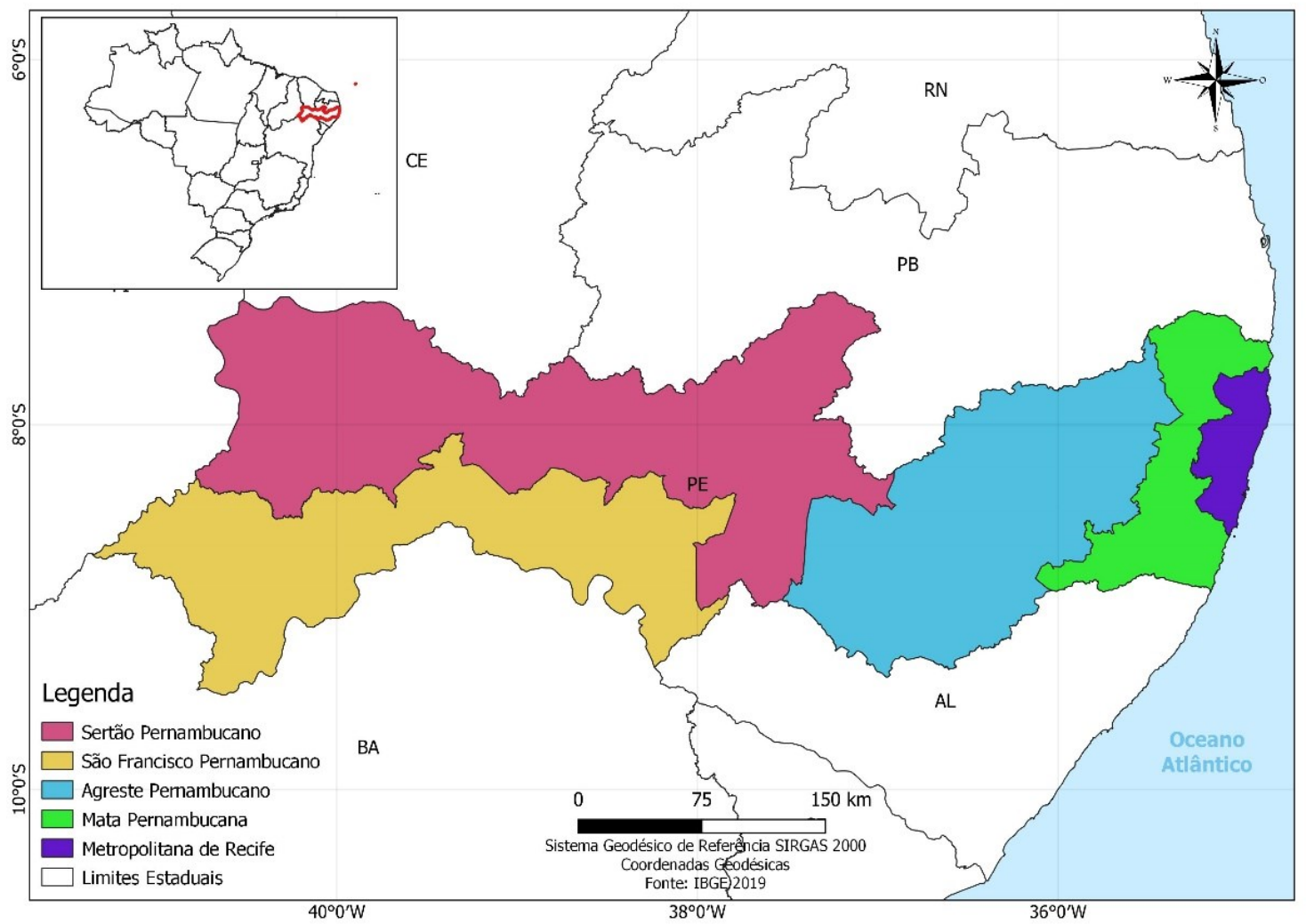


Os dados de violência contra mulher foram obtidos do Sistema de Informação de Agravos de Notificação (Sinan), a partir das fichas de notificação individual de violência, excluindo-se os registros de violência autoinfligida $(n=7.124)$. Os homicídios de mulheres contemplaram todos os registros codificados como causas externas de morbidade e de mortalidade (códigos X85 a Y09 - Agressões), da Classificação Estatística Internacional de Doenças e Problemas Relacionados à Saúde (CID-10), no Sistema de Informações sobre Mortalidade (SIM). Os feminicídios tiveram como fonte o relatório estatístico do Sistema de Informações Policiais (INFOPOL) da Secretaria de Defesa Social de Pernambuco. Foram incluídas as projeções intercensitárias disponibilizadas pelo Instituto Brasileiro de Geografia e Estatística (IBGE) para definição da população do sexo feminino residente no Estado.

Foram calculadas as taxas de violência contra mulher, de homicídios e feminicídios por ano e município. Todos os indicadores foram normalizados pela população feminina do município a cada ano e multiplicado por 100 mil, como segue-se abaixo:

$\operatorname{Taxa}_{\mathrm{ijk}}=\frac{\mathrm{X}_{\mathrm{ijk}}}{\text { Popik }_{\mathrm{ik}}}$

onde i representa o ano de referência ( $i=2016,2017, \ldots, 2019)$, j o tipo de indicador sendo avaliado ( $j$ $=\{$ taxa de violência contra mulher, taxa de homicídios e taxa de feminicídios, $\}$, Pop $p_{i k}$ a população feminina do município $k$ ( $k$ é o município em análise).

Foram avaliadas as tendências temporais de cada indicador $\mathrm{j}$ no período investigado. Para tal, utilizou-se o método dos mínimos quadrados ordinários (regressão linear simples) para estimar retas de tendência. Tais retas tiveram o seguinte formato:

$T a x a_{i j k}=\beta_{j k}+\alpha_{j k} T e m p o_{i j k}+\epsilon_{i j k^{x}}$

O termo de interesse nesta análise foi o parâmetro $\alpha_{j k}$, o qual representa a tendência para cada indicador e município. A interpretação deste parâmetro é a seguinte:

Quando $\alpha_{j k}<0$, existe uma tendência decrescente;

Quando $\alpha_{j k}>0$, existe uma tendência crescente;

Quando $a_{j k} \simeq 0$, não existe tendência. A série é estacionária.

Considerando o total de municípios do estado, foram estimadas 555 tendências. Todos os cálculos e gráficos foram feitos por meio da linguagem de programação $R$ versão 4.0.3.

O estudo utilizou dados secundários, de domínio público, sem identificação dos sujeitos e foi dispensado de apreciação por um Comitê de Ética em Pesquisa envolvendo Seres Humanos.

\section{RESULTADOS}

No estado de Pernambuco foram notificados 33.289 casos de violência contra mulher no período de analisado. Para a taxa de violência contra mulher, a tendência média foi de 18,29 por 100 mil mulheres, com mediana 7,86 por 100 mil mulheres (Tabela 1). Na análise da tendência da taxa de violência contra mulher, observou-se que 142 (76,8\%) dos municípios analisados tiveram tendência crescente, com predomínio na mesorregião do Agreste (Figura 2A e Figura $3 \mathrm{~A}$ ).

Foram registrados 1.038 homicídios femininos, com tendência média de $-0,49$ e a mediana de 0 , indicando que grande parte das tendências é estacionária (Figura 2B). Observou-se que 83 (44,9\%) dos municípios apresentaram estabilidade (Tabela 1 e Figura 3B). Foram registrados 319 feminicídios no estado, no período do estudo. Para a taxa de feminicídios, a tendência média foi de $-0,54$ e a mediana de 0, com $113(61,1 \%)$ apresentando tendência estacionária (Figura 2C e Figura 3C). 
Tabela 1 - Estatísticas descritivas das tendências estimadas para cada município do estado de Pernambuco e indicador (2016-2019).

\begin{tabular}{lrrr}
\hline & \multicolumn{3}{c}{ Indicadores } \\
\cline { 2 - 4 } \multicolumn{1}{c}{ Estatística } & $\begin{array}{c}\text { Taxa de } \\
\text { violência } \\
\text { contra } \\
\text { mulher }\end{array}$ & $\begin{array}{c}\text { Taxa de } \\
\text { homicídios } \\
\text { femininos }\end{array}$ & $\begin{array}{c}\text { Taxa de } \\
\text { feminicídios }\end{array}$ \\
\hline Mínimo & $-122,02$ & $-10,34$ & $-10,34$ \\
1º quartil $_{\text {Média }}$ & 1,31 & $-1,78$ & $-1,00$ \\
Mediana & 18,29 & $-0,54$ & $-0,49$ \\
30 quartil $^{\text {Máximo }}$ & 7,86 & 0,00 & 0,00 \\
Desvio padrão & 23,69 & 0,79 & 0,00 \\
Quantidade de tendência positiva & 192,23 & 7,25 & 5,96 \\
Quantidade de tendência negativa & 34,99 & 3,08 & 2,24 \\
Quantidade de tendência estacionária & 142 & 42 & 25 \\
\% Tendência positiva & 24 & 60 & 47 \\
\% Tendência negativa & 19 & 83 & 113 \\
\% Tendência estacionária & 76,8 & 22,7 & 13,5 \\
\hline
\end{tabular}

Fonte: Elaboração própria a partir dos dados do Sistema de Informação de Agravos de Notificação (Sinan), Sistema de Informações sobre Mortalidade (SIM) e Sistema de Informações Policiais (INFOPOL) da Secretaria de Defesa Social (SDS) de Pernambuco. 
Figura 2 - Estimativas de tendência temporal da violência contra mulher (A), homicídios femininos (B) e feminicídios (C) para cada município de Pernambuco (2016-2019).

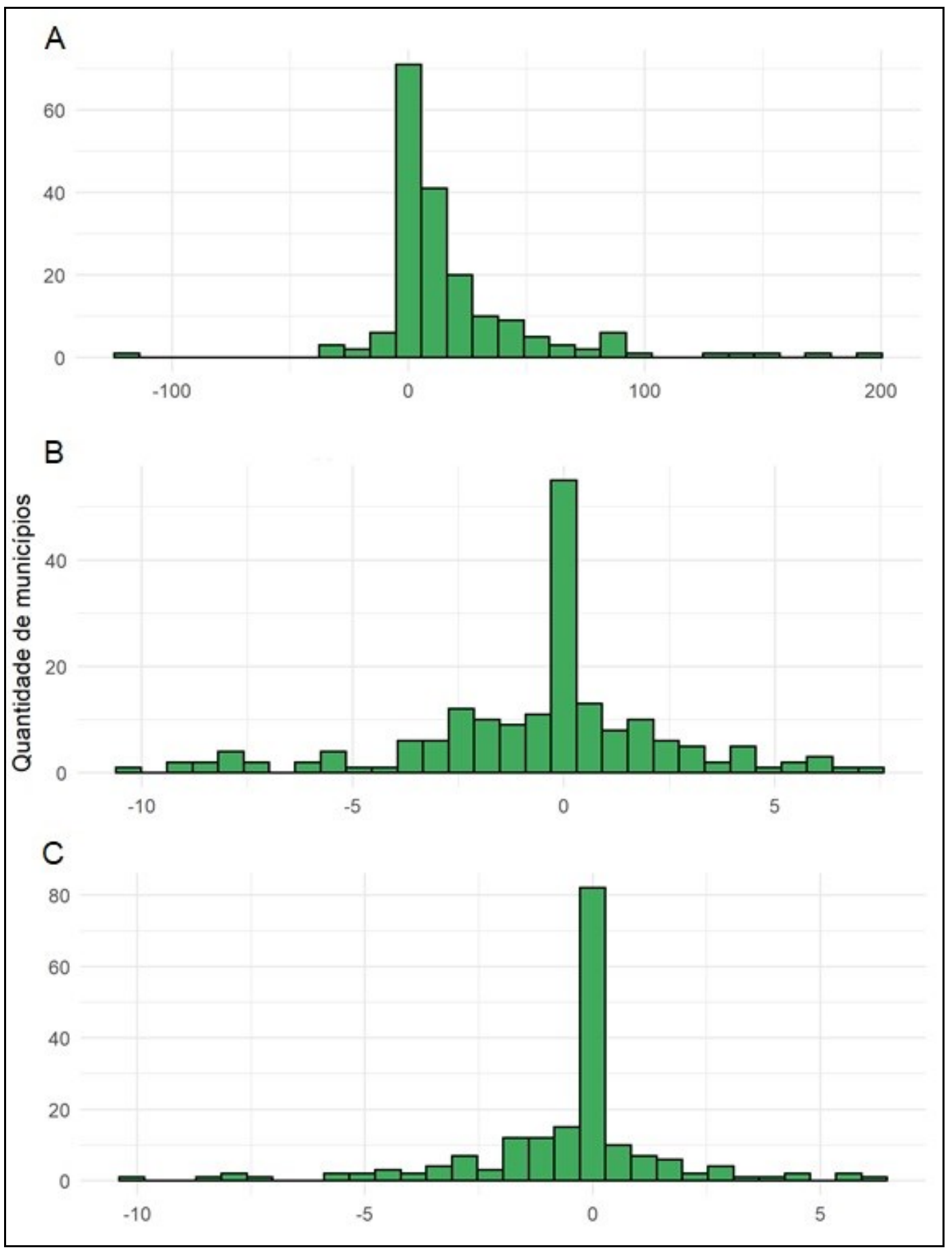

Fonte: Elaboração própria a partir dos dados do Sistema de Informação de Agravos de Notificação (Sinan), Sistema de Informações sobre Mortalidade (SIM) e Sistema de Informações Policiais (INFOPOL) da Secretaria de Defesa Social (SDS) de Pernambuco. 
Figura 3 - Análise da tendência temporal da violência contra mulher (A), homicídios femininos (B) e feminicídios (C) por mesorregião de Pernambuco (2016-2019).

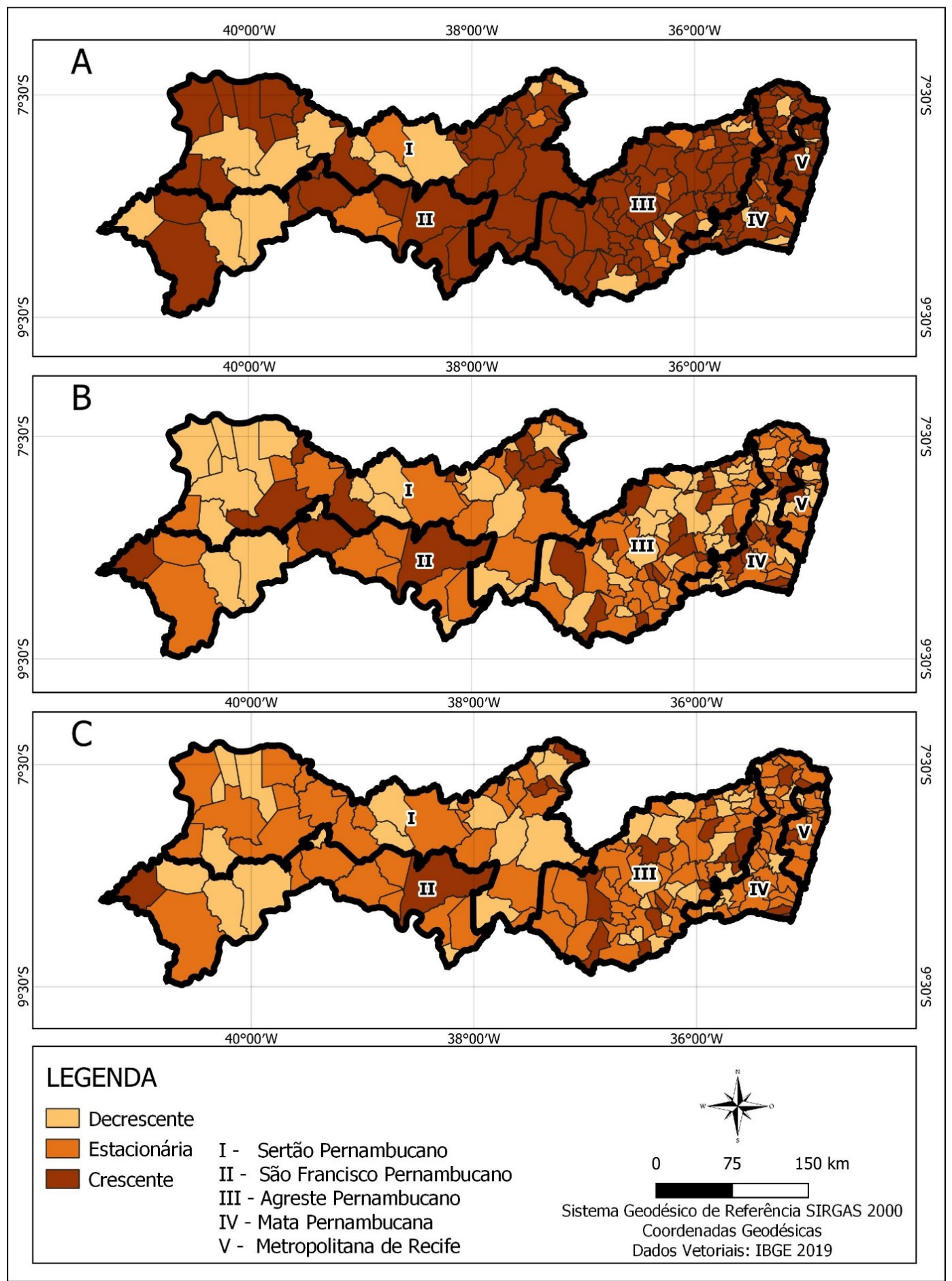

Fonte: Elaboração própria a partir dos dados do Sistema de Informação de Agravos de Notificação (Sinan), Sistema de Informações sobre Mortalidade (SIM) e Sistema de Informações Policiais (INFOPOL) da Secretaria de Defesa Social (SDS) de Pernambuco. 
Da ocorrência em vida à morte: padrões espaciais da violência contra a mulher em Pernambuco
Emylle Thais Melo dos Santos

Conceição Maria de Oliveira

Dayane da Rocha Pimentel

Sheyla Carvalho de Barros

Amanda Priscila de Santana Cabral Silva

Cristine Vieira do Bonfim

\section{DISCUSSÃO}

O estudo demonstrou um aumento da tendência da violência contra mulher e estabilidade nos homicídios femininos e feminicídios no estado de Pernambuco durante o período analisado. A mesorregião do Agreste apresentou maior tendência crescente de violência não letal e letal contra a mulher.

A tendência da violência contra mulher nesse estudo foi crescente para a maior parte dos municípios. De forma similar, pesquisa que analisou a tendência da violência física no Brasil, demonstrou aumento significativo na tendência da violência contra as mulheres (CAVALCANTE et al., 2018). Estudo que analisou as características epidemiológicas e espaciais da violência física em uma cidade do Brasil, identificou que 8 a cada 10 vítimas de violência doméstica eram mulheres, em adição a distribuição espacial apontou os bairros de baixos rendimentos como focos dessa violência (BARBOSA et al., 2019). A vulnerabilidade das mulheres, está diretamente relacionada às desigualdades impostas pela sociedade, tais como acesso à educação, saúde e oportunidades de trabalho (WHO, 2009).

Apesar da tendência de crescimento da taxa de violência contra mulher no estado, devem ser consideradas as subnotificações estar subnotificados devido à naturalização da violência pela sociedade, o despreparo dos serviços no recebimento das vítimas e o medo de denunciar o agressor (ASLAN et al., 2019). Muitas vítimas receiam expor a agressão sofrida por várias razões, entre elas a dependência econômica, medo, vergonha, crenças culturais, culpabilização, entre outros (CARRETTA; BURGESS; DEMARCO, 2016; FERNÁNDEZ-FONTELO et al., 2019). Além disso, a representação do poder do homem sobre a mulher construída e naturalizada historicamente, estão permeadas nas instituições públicas contribuindo para o não tratamento com o devido rigor técnico e legal necessário, a marginalização e a invisibilidade dos casos (BARUFALDI et al., 2017; OLIVEIRA et al., 2019).

As taxas de violência contra mulher encontram-se distribuídas na maioria dos munícipios, com destaques aqueles situados nas macrorregiões do Agreste e Sertão. Alguns estudos relataram maiores taxas de agressão em municípios mais distantes da região metropolitana, o que pode ser justificado pela desigualdade, dependência financeira, escolaridade, refletindo a vulnerabilidade dessas mulheres (BARBOSA et al., 2019; IREGUI-BOHÓRQUEZ; RAMÍREZ-GIRALDO; TRIBÍNURIBE, 2019). Outros fatores devem ser ponderados, como a insuficiência de serviços especializados nessas localidades e à dificuldade de acessar os serviços protetivos como delegacias de mulheres, centros de referência, juizados especiais e casas abrigo (CAMPOS, 2015; EDWARDS, 2015). As instituições que fazem parte da rede de enfrentamento são desigualmente distribuídas, sendo boa parte situadas nas capitais e regiões metropolitanas do Brasil, dificultando a uniformidade do combate à violência de gênero (BARBOSA et al., 2019). Estudo que analisou os efeitos das delegacias da mulher no Brasil sobre os homicídios femininos, identificou que o estabelecimento desse tido de delegacia em um município metropolitano está associado a uma redução na taxa de homicídio feminino em 1,23 mortes por 100.000 mulheres de 15 a 49 anos (uma redução de aproximadamente $17 \%$ na taxa de homicídio feminino em municípios metropolitanos) (PEROVA; REYNOLDS, 2017). Essa redução ainda foi maior para a faixa etária de 15 a 24 anos, A redução da taxa de homicídios de mulheres de 15 a 24 anos foi ainda maior: 5,57 mortes por 100.000 mulheres. As melhores oportunidades econômicas e normas sociais menos tradicionais nas áreas metropolitanas podem explicar os impactos dessas delegacias (PEROVA; REYNOLDS, 2017).

Para os homicídios de mulheres observou-se tendência estacionária, comportamento diferente do encontrado em outras áreas do Brasil. Souza et al. (2017) afirmam que a tendência estacionária deve ser analisada com cautela, considerando os eventos cuja intenção é indeterminada, que pode estar contribuindo para subestimar as taxas de homicídios de mulheres. A análise temporal dos homicídios de mulheres nas regiões do país, evidenciou tendência ascendente para todas as regiões, exceto a Sudeste. Além do risco de feminicídio para as mulheres das coortes de nascimento a partir de 1960 em todas as regiões (SOUZA et al., 2017). Pesquisa que analisou a taxa de mortalidade de mulheres por armas de fogos no país, mostrou aumento nas faixas etárias jovens, que tinham de 4 a 7 anos de escolaridade, solteiras, da raça/cor parda (JUSTINO et al., 2021). De fato, desigualdade e pobreza são fatores que influenciam os homicídios no Brasil (WANG et al., 2016). 
Os homicídios de mulheres são mais frequentes em locais que apresentam maior vulnerabilidade social, nos quais há elevada ocorrência de violência interpessoal, envolvimento com o narcotráfico, uso de álcool, pobreza, entre outros (SOUZA et al., 2017; MEIRA et al., 2019; JUSTINO; COSTA; ANDRADE, 2021).

$\mathrm{Na}$ análise espacial de feminicídios, foi identificada tendência estacionária, no entanto algumas áreas apresentaram tendência crescente de mortalidade por razão de gênero, principalmente no Agreste, as diversidades presentes entre as macrorregiões podem influenciar no número dos homicídios, relacionadas com a aceitação cultural da violência contra a mulher assim como a sua ocorrência (GARCIA e SILVA, 2016). Em uma análise temporal realizada no território brasileiro entre os anos de 2004 a 2015, evidenciou que as taxas de feminicídio aumentaram 4,8\% de 2004 a 2015, com predomínio nas regiões do Norte $(+109,4 \%)$ e Nordeste $(+47,1 \%)$ (MARTINS-FILHO et al., 2018). Uma avaliação realizada em Manaus (AM) constatou que 38\% dos homicídios de mulheres eram feminicídios (ORRELANA et al. 2019). Em Taiwan, um estudo realizado baseado em autópsias, verificou que a proporção de homicídios por razões de gênero era de $31 \%$, representando uma importante fonte de mortalidade prematura entre as mulheres, bem como os impactos para as famílias sobreviventes (FONG et al., 2016).

Em Pernambuco, os feminicídios foram mais frequentes nas áreas onde a violência também é elevada, assim como apontou o estudo desenvolvido no estado do Rio Grande do Sul (LEITES, MENEGHEL e HIRAKATA, 2014). Uma análise realizada em todo território brasileiro evidenciou a heterogeneidade espacial das taxas de feminicídio, semelhante aos dados encontrados (LEITE et al., 2017). Na Colômbia, uma pesquisa desenvolvida em Antioquia verificou que os feminicídios incidiram em distintas unidades geográficas e, como produto dos resultados observados, foi fornecido recomendações às políticas para a enfrentamentos e prevenções específicas (SEPÚLVEDA MURILLO et al., 2018). A incorporação de técnicas de geoprocessamento e análise espacial podem contribuir não apenas na determinação do local de ocorrência de maior incidência, mas também na avaliação de intervenções destinadas ao enfrentamento deste fenômeno a partir da assistência à saúde, vigilância, entre outros, além de conceber maior visibilidade ao enfrentamento do feminicídio (SEPÚLVEDA MURILLO et al., 2018; PRADO e VIEIRA, 2019).

Considerando que a violência contra a mulher é um problema complexo, a utilização das múltiplas fontes de dados disponíveis empregadas nesta pesquisa possibilitou a mensuração e monitoramento do fenômeno. A análise espacial evidenciou que a violência contra mulher durante os anos analisados incidiu em todo o espaço geográfico. Um estudo que verificou a distribuição espacial da violência doméstica no município de João Pessoa (PB) constatou que a violência de gênero também esteve presente em todo o espaço geográfico (OLIVEIRA et al., 2019). Diante do aumento da violência, se faz necessário refletir sobre os determinantes sociais e situações de vulnerabilidade que contribuem com o crescente aumento das taxas, incluindo baixa escolaridade, renda, religião, estado civil, entre outros, com o objetivo de desenvolver estratégias intersetoriais para o enfrentamento da violência de gênero (ABOLFOTOUH; ALMUNEEF, 2019; ABRUNHOSA et al., 2020).

A redução da violência contra mulher depende de um conjunto de ações que precisam ser desenvolvidas tais como: formação e treinamento dos profissionais da atenção primária à saúde para identificação de casos de violência contra mulher; ampliação do quantitativo e distribuição espacial das casas de abrigo e de delegacias especializadas da mulher; aprimoramento dos dados dos sistemas de informações que podem ser empregados para as medidas prevenção da violência contra mulher; além do cumprimento das medidas protetivas.

Como limitações do estudo, destacam-se: a) o uso de dados secundários que podem ter subnotificações e incompletudes nos registros; b) o uso do município como unidade geográfica de referência, cujos pequenos números podem contribuir para flutuações aleatórias. Apesar do estudo apresentar essas possíveis limitações, o desenho ecológico possibilitou a identificação de áreas geográficas com maiores riscos de violência não letal e letal contra a mulher. Acrescente-se a relevância dos resultados podem contribuir com as estratégias de redução da violência contra mulher. 


\section{CONCLUSÃO}

O presente estudo evidenciou tendência ascendente da violência perpetrada contra a mulher, especialmente na mesorregião do Agreste, possibilitando a identificação de áreas que requerem atenção prioritária para redução da violência. Quanto aos homicídios femininos e feminicídio foi contatado que no período estudado a tendência foi estacionária no estado de Pernambuco.

Neste sentido, evidencia-se a necessidade de melhorias nas políticas públicas do estado de Pernambuco que visam à proteção das mulheres em situação de violência, bem como a ampliação dos serviços especializados para as áreas prioritárias. As medidas protetivas de urgência no combate à violência doméstica previstas pela legislação brasileira devem ser fortalecidas, contribuindo para a prevenção e o enfrentamento das agressões, assim como a redução dos casos de feminicídio. $O$ combate à violência de gênero requer a participação de diferentes setores da sociedade, garantindo uma melhor qualidade de vida para as mulheres.

\section{REFERÊNCIAS}

ABOLFOTOUH, M. A.; ALMUNEEF, M. Prevalence, pattern and factors of intimate partner violence against Saudi women. Journal of Public Health, p. 1-9, 2019.

https://doi.org/10.1093/pubmed/fdz092

ABRUNHOSA, C.; RODRIGUES, A. C.; CRUZ, A. R.; GONÇALVES, R. A.; CUNHA, O. Crimes Against Women: From Violence to Homicide. Journal of Interpersonal Violence, p. 1-24, 2020. https://doi.org/10.1177/0886260520905547

ASLAN, E.; BODUR, G.; BEJI, N. K.; ALKAN, N.; AKSOY, Ö. Exposure to domestic violence in women living in istanbul and aegean regions: A turkish sample. Ciencia e Saude Coletiva, v. 24, n. 8, p. 2835-2844, 2019. https://doi.org/10.1590/1413-81232018248.22952017

BARBOSA, K. G. N.; WALKER, B. B.; SCHUURMAN, N.; CAVALCANTI, S. D. L. B.; FERREIRA E FERREIRA, E.; FERREIRA, R. C. Epidemiological and spatial characteristics of interpersonal physical violence in a Brazilian city: A comparative study of violent injury hotspots in familial versus non-familial settings, 2012-2014. PLoS ONE, v. 14, n. 1, p. 1-19, 2019.

https://doi.org/10.1371/journal.pone.0208304

BARUFALDI, L. A.; SOUTO, R. M. C. V.; CORREIA, R. S. B.; MONTENEGRO, M. M. S.; PINTO, I. V.; SILVA, M. M. A.; LIMA, C. M. Violência de gênero: comparação da mortalidade por agressão em mulheres com e sem notificação prévia de violência. Ciência e Saude Coletiva, v. 22, n. 9, p. 2929 2938, 2017. https://doi.org/10.1590/1413-81232017229.12712017

BRASIL. Lei no 13.104, de 9 de março de 2015. Altera o art. 121 do Decreto-Lei no 2.848, de 7 de dezembro de 1940 - Código Penal, para prever o feminicídio como circunstância qualificadora do crime de homicídio, e o art. $1^{\circ}$ da Lei $n^{\circ} 8.072$, de 25 de julho de 1990, para incluir o feminicídio no rol dos crimes hediondos. Diário Oficial da União, 2015.

CAMPOS, C. H. Desafios na implementação da Lei Maria da Penha. Revista Direito GV, São Paulo, v. 11, n. 2, p. 391-406, 2015.

https://doi.org/10.1590/1808-2432201517

CARRETTA, C. M.; BURGESS, A. W; DEMARCO, R. To Tell or Not to Tell. Violence Against Women, v. 22, n. 13, p. 1499-1518, 2016. https://doi.org/10.1177/1077801215627507

EDWARDS, K. M. Intimate Partner Violence and the Rural-Urban-Suburban Divide: Myth or Reality? A Critical Review of the Literature. Trauma, Violence, and Abuse, v. 16, n. 3, p. 359-373, 2015. https://doi.org/10.1177/1524838014557289

FERNÁNDEZ-FONTELO, A.; CABAÑA, A.; JOE, H.,; PUIG, P.; MORIÑA, D. Untangling serially dependent underreported count data for gender-based violence. Statistics in Medicine, v. 38, p. 4404- 4422, 2019. https://doi.org/10.1002/sim.8306 
FERNÁNDEZ-GONZÁLEZ, L.; CALVETE, E.; ORUE, I. Women victims of gender violence in shelters: Sociodemographic and maltreatment characteristics. Psychosocial Intervention, v. 26, n. 1, p. 9-17, abr. 2017. https://doi.org/10.1016/j.psi.2016.10.001

FONG, W. L.; PAN, C. H.; LEE, J. C.; LEE, T. T.; HWA, H. L. Adult femicide victims in forensic autopsy in Taiwan: A 10-year retrospective study. Forensic Science International. V. 266, p. 80-85, 2016. https://doi.org/10.1016/j.forsciint.2016.05.008

FONTES, K. B.; ALARCÃO, A. C. J.; NIHEI, O. K.; PELLOSO, S. M.; ANDRADE, L.; CARVALHO, M. D. B. Regional disparities in the intimate partner sexual violence rate against women in Paraná State, Brazil, 2009-2014: an ecological study. BMJ Open, 8:e018437, 2018.

FÓRUM BRASILEIRO DE SEGURANÇA PÚBLICA. Anuário Brasileiro de Segurança Pública 2019. 2019.

GARCIA, L. P.; SILVA, G. D. M. DA. Mortalidade de mulheres por agressões no Brasil: perfil e estimativas corrigidas (2011 - 2013). Instituto de Pesquisa Econômica Aplicada - Ipea, 2016.

GARCIA, L. P.; DA SILVA, G. D. M. Violência por parceiro íntimo: Perfil dos atendimentos em serviços de urgência e emergência nas capitais dos estados brasileiros, 2014. Cadernos de Saúde Publica, v. 34, n. 4, 2018. https://doi.org/10.1590/0102-311x00062317

GOMES, I. S. Feminicídios: um longo debate. Revista Estudos Feministas, v. 26, n. 2, p. 1-16, 2018. https://doi.org/10.1590/1806-9584-2018v26n239651

ILANZO, M. P. Q.; URBANO, O. M. C.; DELGADO, M. C.; RAMIREZ, N. P.; MENDOZA, G; M. P.; GARCIA, A. E. O. Violencia extrema contra la mujer y feminicidio en el Perú. Revista Cubana de Salud Pública, v. 44, n. 2, p. $263-276$, jun. 2018.

INGRAM, M. C; COSTA, M. M. A Spatial Analysis of Homicide Across Brazil's Municipalities. Homicide Studies, v. 21, n. 2, p. 87-110, 2016. https://doi.org/10.1177/1088767916666603

Instituto de Pesquisa Econômica Aplicada (IPEA). Fórum Brasileiro de Segurança Pública. Atlas da Violência. 2019. Disponível em: <http://www.forumseguranca.org.br/wp-

content/uploads/2019/06/Atlas-da-Violencia-2019 05jun vers\%C3\%A3o-coletiva.pdf>. Acesso em: 19 set. 2020.

IREGUI-BOHÓRQUEZ, A. M.; RAMÍREZ-GIRALDO, M. T.; TRIBÍN-URIBE, A. M. Domestic Violence Against Rural Women in Colombia: The Role of Labor Income. Feminist Economics, v. 25, n. 2, p. 146-172, 2019. https://doi.org/10.1080/13545701.2019.1566752

JUSTINO, D. C. P.; COSTA, K. T. D. S.; ANDRADE, F. B. Epidemiological profile of female firearmrelated mortality. Medicine (Baltimore), v. 100, n. 2, 2021.

https://doi.org/10.1097/MD.0000000000024222

LEITE, F. M. C.; MASCARELLO, K. C.; ALMEIDA, A. P. S. C.; FÁVERO, J. L.; SANTOS, A. S.; SILVA, I. C. M.; WEHRMEISTER, F. C. Análise da tendência da mortalidade feminina por agressão no Brasil , estados e regiões. Ciência \& Saúde Coletiva, v. 22, n. 9, p. 2971-2978, 2017. https://doi.org/10.1590/1413-81232017229.25702016

LEITE, F. M. C.; LUIS, M. A.; AMORIM, M. H. C.; MACIEL, E. L. N.; GIGANTE, D. P. Violência contra a mulher e sua associação com o perfil do parceiro íntimo: estudo com usuárias da atenção primária. Revista brasileira de epidemiologia, v. 22, n. 60530812, p. e190056, 2019.

https://doi.org/10.1590/1980-549720190056

LEITES, G. T.; MENEGHEL, S. N.; HIRAKATA, V. N. Homicídio feminino no Rio Grande do Sul, Brasil. Revista brasileira de epidemiologia, São Paulo, v. 17, n. 3, pág. 642-653, 2014. https://doi.org/10.1590/1809-4503201400030006

LUCENA, K. D.; SILVA, A. T.; MORAES, R. M.; SILVA, C. C.; BEZERRA, I. P. Spatial analysis of domestic violence against women from 2002 to 2005 in João Pessoa, Paraíba State, Brazil.

Cadernos de Saúde Pública, v. 28, p. 1111-21, 2012. https://doi.org/10.1590/S0102$\underline{311 \times 2012000600010}$ 
MASCARENHAS, M. D. M.; TOMAZ, G. R.; MENESES, G. M. S.; RODRIGUES, M. T. P.; PEREIRA, V. O. M.; CORASSA, R.B. Análise das notificações de violência por parceiro íntimo contra mulheres, Brasil, 2011-2017. Revista Brasileira de Epidemiologia [online], v. 23, n. Suppl 01, 2020. https://doi.org/10.1590/1980-549720200007.supl.1

MARGARITES, A. F.; MENEGHEL, S. N.; CECCON, R. F. Feminicídios na cidade de Porto Alegre: Quantos são? Quem são?. Revista Brasileira de Epidemiologia, v. 20, n. 2, p. 225-236, abr.-jun. 2017. https://doi.org/10.1590/1980-5497201700020004

MARTINS-FILHO, P. R. S.; MENDES, M. L. T.; REINHEIMER, D. M.; NASCIMENTO-JÚNIOR, E. M.; VAEZ, A. C.; SANTOS, V. S.; SANTOS, H. P. Femicide trends in Brazil: relationship between public interest and mortality rates. Archives of Women's Mental Health, v. 21, p. 579-582, 2018. https://doi.org/10.1007/s00737-018-0842-1

MEIRA, K. C.; COSTA, M. A. R.; HONÓRIO, A. C. C.; SIMÕES, T. C.; CAMARGO, M. P.; SILVA, G. W. S. Temporal trend of the homicide rate of Brazilian women. Revista da Rede de Enfermagem do Nordeste, v. 20, p. e39864, 2019. https://doi.org/10.15253/2175-6783.20192039864

MENEGHEL, S. N.; PORTELLA, A. P. Feminicídios: Conceitos, tipos e cenários. Ciencia e Saude Coletiva, v. 22, n. 9, p. 3077-3086, 2017. https://doi.org/10.1590/1413-81232017229.11412017

OLIVEIRA, B. M.; LUCENA, K. D. T.; GOMES, R. G. S.; COÊLHO, H. F. C.; VIANNA, R. P. T.; MEIRA, R. M. B. Spatial distribution of domestic violence against women. Journal of Human Growth and Development, São Paulo, v. 29, n. 1, p. 102-109, abr. 2019.

https://doi.org/10.7322/ihgd.152305

PENGPID, S.; PELTZER, K.; LAOSEE, O.; SUTHISUKON, K. Intimate partner sexual violence and risk for femicide, suicidality and substance use among women in antenatal care and general outpatients in Thailand. BMC Women's Health, v. 18, n. 1, p. 1-7, 2018. https://doi.org/10.1186/s12905018-0526-z

PEROVA, E.; REYNOLDS, S. A. Women's police stations and intimate partner violence: Evidence from Brazil. Social Science \& Medicine, v. 174, p. 188-196, 2017.

https://doi.org/10.1016/j.socscimed.2016.12.008

PRADO, A. M.; VIEIRA, C. V. Análise espacial de risco para leptospirose no município de Joinville (SC). Hygeia - Revista Brasileira de Geografia Médica e da Saúde, v. 15, n. 34, p. 85 - 101, 27 dez. 2019. https://doi.org/10.14393/Hygeia153449520

RIBEIRO, M. I. C.; SILVA, M. G. S. N. Violência, Vulnerabilidade e Desigualdade Socioespacial: Análise das Dificuldades Enfrentadas pelas Mulheres Vítimas de Violência Doméstica na Área Urbana de Porto Velho - RO. Revista Latino-americana de Geografia e Gênero, v. 6, p. 92-104, 2015. https://doi.org/10.5212/Rlagg.v.6.i2.0006

SAN SEBASTIÁN, M.; VIVES-CASES, C.; GOICOLEA, I. "Closer to the Unfair Reality": Magnitude and Spatial Analysis of Femicides in Ecuador. Journal of Interpersonal Violence, p. 1-12, 2019. https://doi.org/10.1177/0886260519863721

SANDOVAL, G. A.; MARINHO, F.; DELANEY, R.; PINTO, I. V.; LIMA, C. M. D.; COSTA, R. M.; BELLO-CORASSA, R.; PEREIRA, V. O. M.; STEVENS, A. Mortality risk among women exposed to violence in Brazil: a population-based exploratory analysis. Public Health, v. 179, p. 45-50, 2020. https://doi.org/10.1016/j.puhe.2019.09.019

SANZ-BARBERO, B.; BARÓN, N.; VIVES-CASES, C. Prevalence, associated factors and health impact of intimate partner violence against women in different life stages. PLoS ONE, v. 14, n. 10, p. 1-12, 2019. https://doi.org/10.1371/journal.pone.0221049

SEPÚLVEDA MURILLO, F. H.; OLMO, J. C.; CORTÁZAR, A. R. G. The spatial heterogeneity of factors of feminicide: The case of Antioquia-Colombia. Applied Geography, p. 63-73, 2018. https://doi.org/10.1016/i.apgeog.2018.01.006

SILVA, G. C. B.; NOBREGA, W. F. S.; MELO NETO, O. M.; SOARES, R. S. C.; OLINDA, R. A.; D'AVILA, S. L. B. C; CAVALCANTI, A. L. Distribuição espacial e perfil epidemiológico das notificações 
da violência contra a mulher em uma cidade do nordeste brasileiro. Arch Health Invest, v. 8, n. 19, p. 580-585. 2019. https://doi.org/10.21270/archi.v8i10.3814

SOUZA, E. R.; MEIRA, K. C.; RIBEIRO, A. P.; SANTOS, J.; GUIMARÃES, R. M.; BORGES, L. F.; OLIVEIRA, L. V.; SIMÕES, T. C. Homicídios de mulheres nas distintas regiões brasileiras nos últimos 35 anos: análise do efeito da idade-período e coorte de nascimento. Ciência \& Saúde Coletiva [online], v. 22, n. 9, 2-17. https://doi.org/10.1590/1413-81232017229.12392017

WAISELFISZ, J. J. Mapa da violência 2015: homicídio de mulheres no Brasil. Flacso Brasil, ago. 2015. Disponível em:<http://www.mapadaviolencia.org.br/>. Acesso em: 19 set. 2020.

WANG, H.; NAGHAVI, M.; ALLEN, C.; et al. GBD 2015 Mortality and causes of death collaborators. Global, regional, and national life expectancy, all-cause-specific mortality for 249 causes of death, 1980-2015: a systematic analysis for the Global Burden of Disease Study 2015. The Lancet, v. 388, n. 10053, p. 1459-544, 2016.

WORLD HEALTH ORGANIZATION (WHO). Promoting gender equality to prevent violence against women. Geneva, 2009. Disponível em: <https://apps.who.int/iris/bitstream/handle/10665/44098/9789241597883_eng.pdf>. Acesso em: 01 jun. 2021.

WORLD HEALTH ORGANIZATION (WHO). Violence against women. Geneva, 2021. Disponível em: <https://www.who.int/news-room/fact-sheets/detail/violence-against-women>. Acesso em: 20 mai. 2021.

\section{i Fonte de financiamento}

Financiamento público. Coordenação de Aperfeiçoamento de Pessoal de Nível Superior - Brasil (CAPES) código de financiamento 001.

\section{Número de identificação/aprovação do CEP}

O estudo foi realizado de acordo com as recomendações de ética em pesquisa. Foram utilizados dados agregados e sem identificação individual, que dispensa a aprovação por um Comitê de Ética em Pesquisa, conforme a Resolução 510/2016 do Conselho Nacional de Saúde. 